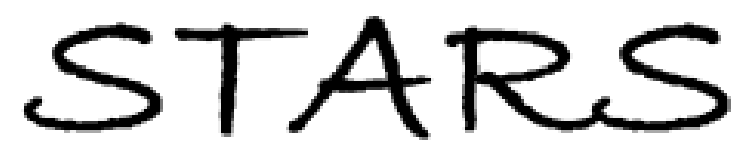

University of Central Florida

STARS

$1-1-2013$

\title{
Simultaneous optical and photoacoustic measurement of nonlinear absorption
}

\section{Panit Chantharasupawong}

University of Central Florida

Reji Philip

Jayan Thomas

University of Central Florida

Find similar works at: https://stars.library.ucf.edu/facultybib2010

University of Central Florida Libraries http://library.ucf.edu

This Article is brought to you for free and open access by the Faculty Bibliography at STARS. It has been accepted for inclusion in Faculty Bibliography 2010 s by an authorized administrator of STARS. For more information, please contact STARS@ucf.edu.

\section{Recommended Citation}

Chantharasupawong, Panit; Philip, Reji; and Thomas, Jayan, "Simultaneous optical and photoacoustic measurement of nonlinear absorption" (2013). Faculty Bibliography 2010s. 3778.

https://stars.library.ucf.edu/facultybib2010/3778

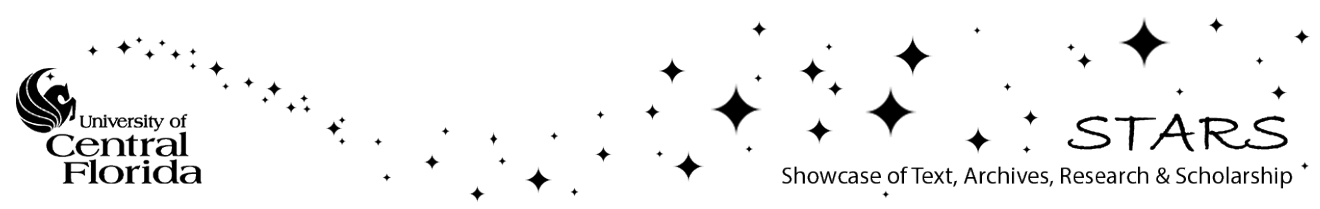




\section{Simultaneous optical and photoacoustic measurement of nonlinear absorption}

Cite as: Appl. Phys. Lett. 102, 041116 (2013); https://doi.org/10.1063/1.4789870

Submitted: 06 December 2012 . Accepted: 16 January 2013 . Published Online: 01 February 2013

Panit Chantharasupawong, Reji Philip, and Jayan Thomas
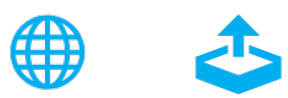

\section{ARTICLES YOU MAY BE INTERESTED IN}

Nonlinear scattering studies of carbon black suspensions using photoacoustic Z-scan technique

Applied Physics Letters 103, 151104 (2013); https://doi.org/10.1063/1.4824448

Photoacoustic imaging in biomedicine

Review of Scientific Instruments 77, 041101 (2006); https://doi.org/10.1063/1.2195024

Laser fluence dependence on emission dynamics of ultrafast laser induced copper plasma

Journal of Applied Physics 120, 185901 (2016); https://doi.org/10.1063/1.4967313

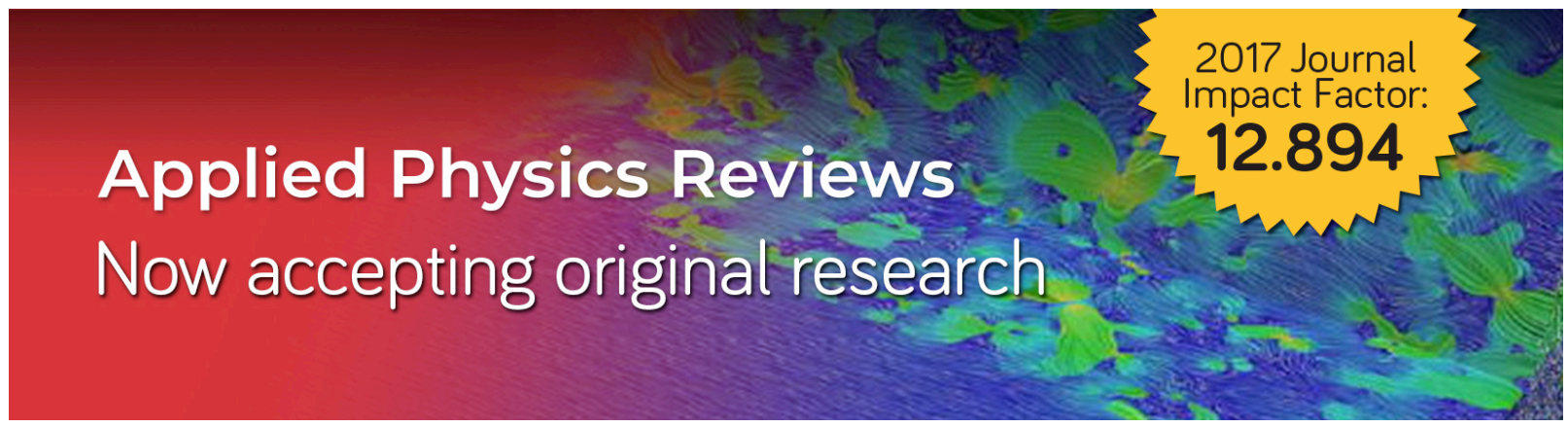




\title{
Simultaneous optical and photoacoustic measurement of nonlinear absorption
}

\author{
Panit Chantharasupawong, ${ }^{1}$ Reji Philip, ${ }^{2, \text { a) }}$ and Jayan Thomas ${ }^{1,2,3, b)}$ \\ ${ }^{1}$ College of Optics and Photonics, CREOL, University of Central Florida, Orlando, Florida 32826, USA \\ ${ }^{2}$ NanoScience Technology Center, University of Central Florida, Orlando, Florida 32826, USA \\ ${ }^{3}$ Department of Material Science and Engineering, University of Central Florida, Orlando, \\ Florida 32826, USA
}

(Received 6 December 2012; accepted 16 January 2013; published online 1 February 2013)

\begin{abstract}
A measurement technique to perform optical Z-scan and photoacoustic Z-scan simultaneously called OPAZ-scan is demonstrated. It is found that the simultaneous measurement of the optical and photoacoustic Z-scan signals provides substantially better insight into the mechanism of optical nonlinearity. The system is able to identify mixed nonlinear processes within a mixture of nonlinear scattering species and nonlinear absorbers. (C) 2013 American Institute of Physics.
\end{abstract}

[http://dx.doi.org/10.1063/1.4789870]

There is a considerable interest in knowing nonlinear optical absorption properties of materials. In applications such as optical limiting for sensor protections ${ }^{1-4}$ and photo thermal cancer therapy, ${ }^{5,6}$ strong nonlinear optical absorbers are desirable. There are several means to measure the nonlinear absorption of materials, for example, transmission, ${ }^{7-9}$ calorimetric, ${ }^{10,11}$ and photoacoustic (PA) ${ }^{12,13}$ measurements. In transmission measurements, change in transmitted optical signal through an absorbing sample is monitored with varied incident intensity. Calorimetric measurements monitor change in temperature, and photoacoustic measurements records generated sound waves due to absorption of materials. The most well adopted technique for measuring nonlinear absorption of both solid and liquid samples is an open aperture Z-scan, ${ }^{14}$ which is a transmission based measurement. ${ }^{15,16}$ In this technique, the sample is scanned through a focused laser beam and the sample transmittances at different positions (Z-position) are noted. Nonlinear absorption coefficients can be calculated from plots of normalized transmission vs. Z-positions. Small changes in light transmission can be easily detected using highly sensitive photodetectors. With this technique, however, samples with high optical transparency and good optical quality are required for performing accurate measurements. In contrast, calorimetric and photoacoustic measurements do not require samples with optical transparency. ${ }^{17}$ Similar to optical Z-scan, a photoacoustic Z-scan (PAZ-scan) technique scans the sample through a focused beam. ${ }^{18}$ However, in PAZ-scan, instead of monitoring transmitted light, acoustic signal is recorded. Moreover, determination of nonlinear absorption parameters of optically opaque materials is possible with this photoacoustic technique. One of the drawbacks of this technique, however, is the low sensitivity in acoustic wave detection since the PA generation efficiency is only in the order of $10^{-12}$ to $10^{-8} .19$

In this paper, we propose a technique which combines the PAZ-scan and conventional optical Z-scan. Photoacoutic

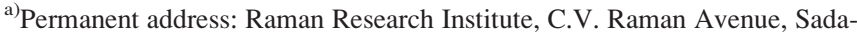
shivanagar, Bangalore 560080, India.

b) Author to whom correspondence should be addressed. Electronic Mail: Jayan.Thomas@ucf.edu.
}

and optical transmission signal are measured and obtained simultaneously in one experimental run. This combined optical and photoacoustic Z-scan technique will be called OPAZscan. The data obtained from both signals are employed to determine nonlinear absorption parameters. We have found that when these two sets of information are collected simultaneously, erroneous conclusions can be avoided. Furthermore, herein, we also demonstrate the advantages of this combined technique.

A Q-switched, frequency-doubled Nd:YAG laser (Minilite I, Continuum) was used to generate $5 \mathrm{~ns}$ (FWHM) pulses at $532 \mathrm{~nm}$. The laser output was spatially filtered to obtain a neat Gaussian beam profile, and focused using a $200 \mathrm{~mm}$ focal length plano-convex lens. A homemade brass PA cell was mounted on a linear translation stage (Newport, ILS150PP). The cell has a diameter of $8 \mathrm{~cm}$ and height of $5 \mathrm{~cm}$. An ultrasonic transducer (Olympus NDT, model A315-SU) and glass windows are fixed on the circular cell wall as shown in Figure 1. The cell has a brass lid with a slotted teflon cap in the center, through which a $1 \mathrm{~mm}$ path length cuvette containing the sample can be inserted. The cuvette was positioned at an angle such that its front surface faces the transducer for better acoustic detection. The cell was filled with water to achieve

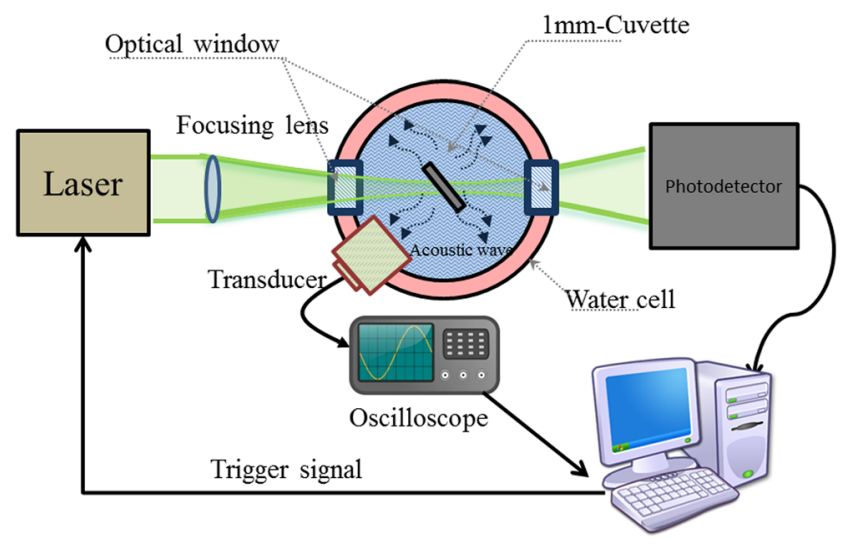

FIG. 1. Schematic of the experimental setup used for simultaneous measurement of optical and photoacoustic Z-scan. A computer program is used for triggering laser output and acquiring data. 
(a)

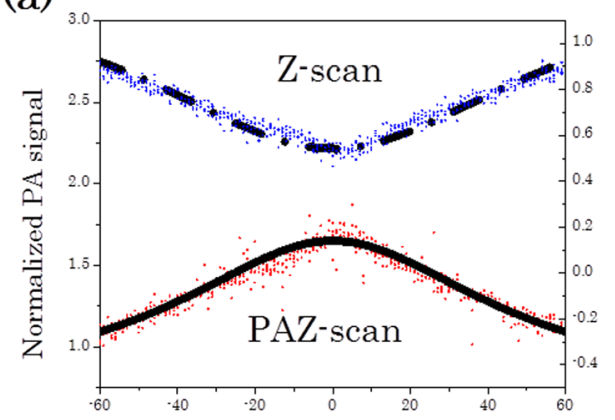

(c)

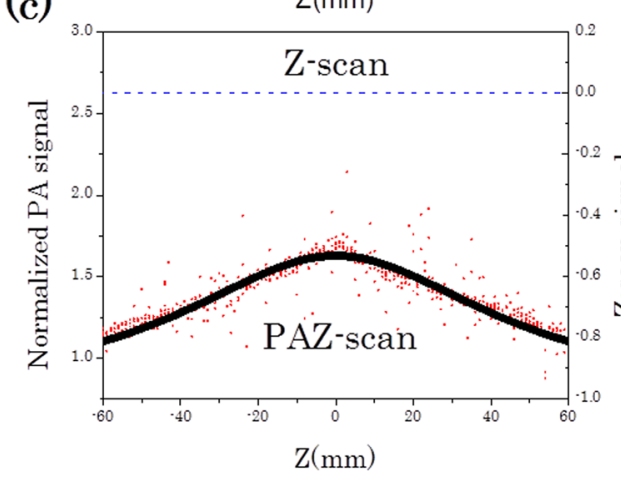

(b)

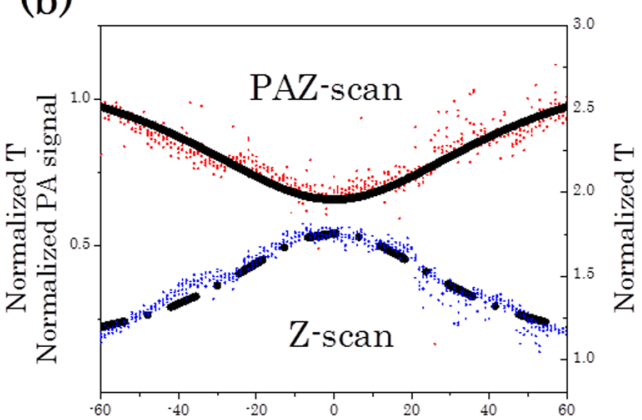

(d)
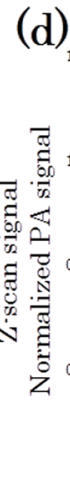

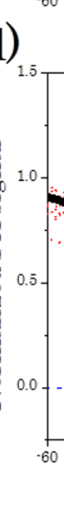

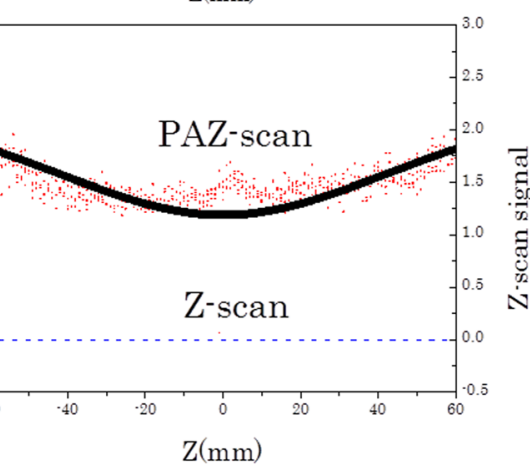

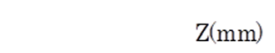

FIG. 2. OPAZ-scan curves of (a) PCBM with $20 \%$ linear transmission and Ein of $112 \mu \mathrm{J}$, (b) DODCI with $20 \%$ linear transmission and Ein of $112 \mu \mathrm{J}$, (c) PCBM with $\sim 0 \%$ linear transmission and Ein of $80 \mu \mathrm{J}$, and (d) DODCI with $\sim 0 \%$ linear transmission and Ein of $128 \mu \mathrm{J}$. Red and blue dots are experimental data for PAZ-scan and Z-scan, respectively. The black solid and dashed lines are the fitted curves.

good acoustic coupling between the sample and the transducer. The acoustic signals were collected in reflection geometry by the transducer which was connected to an oscilloscope. The peak signals at $20 \mu$ s after the incident laser pulse were measured. This delay corresponds to the time required for sound wave to travel through water before reaching the transducer. A pyroelectric energy probe (LaserProbe, RjP-735) was used to measure sample transmission. By fixing the input laser pulse energy (Ein) at a suitable value and translating the cell along the laser beam near the focal region, the incident laser fluence on the sample $(\operatorname{Fin}(z))$ was varied. Maximum fluence is attained at the beam focus $(\mathrm{z}=0)$, and the fluence reduces as a Lorentzian function away from the focal point. The beam radius $w_{0}$ at the focus was measured to be $70 \mu \mathrm{m}$. A computer program was used to run the experiment. Simultaneous plots of peak acoustic signals and optical transmission were generated as a function of sample positions.

The samples used with this experiment were

(i) Saturable absorber: organic dye DODCI (3,3'-diethyloxadicarbocyanine iodide) in methanol, at two different concentrations (with $20 \%$ transmission and $\sim 0 \%$ transmission at $532 \mathrm{~nm}$, respectively).

(ii) Reverse saturable absorber: Funtionalized-C60([6,6]phenyl- $\mathrm{C}_{61}$-butyric acid methyl ester: $\mathrm{PCBM}$ ) in chloroform, at two different concentrations (with $20 \%$ transmission and $\sim 0 \%$ transmission at $532 \mathrm{~nm}$, respectively).

(iii) Scattering sample: carbon-black in water suspension with $1 \%$ transmission at $532 \mathrm{~nm}$.

Normalized transmission and normalized peak PA signals are plotted against sample positions for these samples as shown in Figures 2 and 3. In the case of reverse saturable absorbers (Figure 2(a)), light absorption increases as the incident intensity increases. This gives rise to decrease in transmission and hence the Z-scan signal as the sample is moved toward the focal point. The PA signal, on the other hand, shows a peak since the acoustic generation is proportional to the absorbed energy. On the contrary, saturable absorbers, Figure 2(b), have reversed characteristics because light absorption is less near the focal point. It is good to note here that, even though the OPAZ-scan is demonstrated with high concentration samples, this technique is also applicable with light sample as well. In the case of PCBM, a sample with transmission as high as $90 \%$ can be measured with this technique.

In order to find nonlinear coefficients from transmission signals, the following differential equation for optical intensity loss in thin sample is considered:

$$
\frac{d I}{d z}=-\mu_{a} I,
$$

where $I$ is the optical intensity, $\mathrm{z}$ is the propagation distance, and $\mu_{a}$ is the absorption coefficient described by Eqs. (2) and

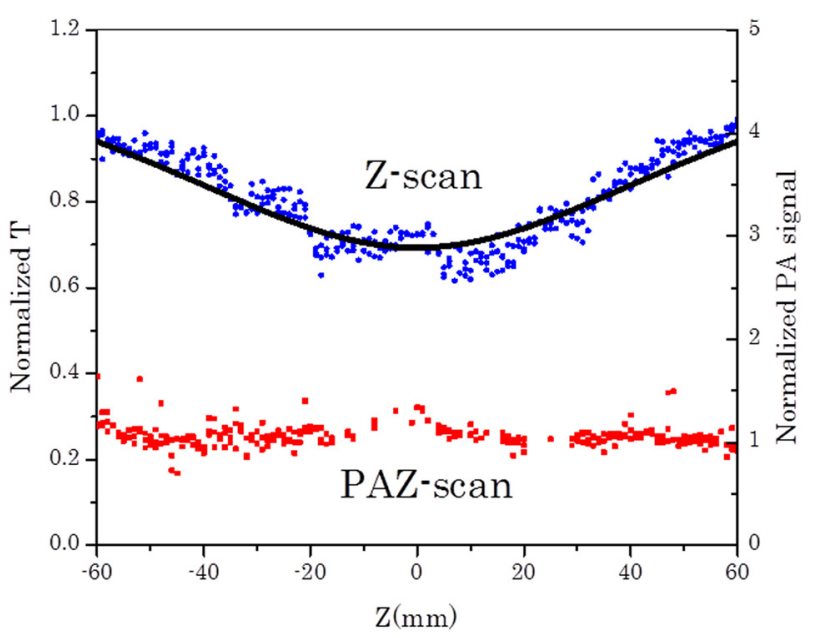

FIG. 3. OPAZ-scan curves of carbon-black dispersion in water with $1 \%$ transmission at $532 \mathrm{~nm}$ and Ein of $103 \mu \mathrm{J}$. The solid black line is a numerical fit $\mathrm{z}$ scan curve. 
(3) for reverse saturable absorber ${ }^{20}$ and saturable absorber, ${ }^{21}$ respectively,

$$
\begin{gathered}
\mu_{a}=\left(\alpha_{0}+\beta_{\text {eff }} I\right), \\
\mu_{a}=\alpha_{0} /\left(1+I / I_{\text {sat }}\right),
\end{gathered}
$$

where $\beta_{\text {eff }}$ is the effective nonlinear absorption coefficient for reverse saturable absorbers and $I_{\text {sat }}$ is the saturation intensity of saturable absorbers. The intensity for a $\mathrm{TEM}_{00}$ Gaussian beam propagating through a sample along the $+\mathrm{z}$ direction is of the form:

$$
I(z, t)=\left[\frac{w_{0}^{2} I_{0}(x, t)}{w^{2}(z)}\right] \exp \left(-\frac{2 r^{2}}{w^{2}(z)}\right),
$$

where $w^{2}(z)=w_{0}^{2}\left(1+z^{2} / z_{0}^{2}\right), \mathrm{z}$ is the sample position relative to the focus, $w_{0}$ is the radius of the beam waist, and $z_{0}=\pi w_{0}^{2} / \lambda$ is the Rayleigh length. By integrating Eq. (1) over sample length $\mathrm{L}$, the optical intensity transmitted through a sample can be determined. For a temporally Gaussian pulse, the normalized transmission of the sample is given by

$$
T_{\text {norm }}(z)=\frac{1}{\sqrt{\pi}} \int_{-\infty}^{\infty} T(z, t) d t,
$$

with

$$
T(z, t)=\frac{\int_{0}^{\infty} I_{\text {out }} r d r}{e^{-\alpha_{0} L} \int_{0}^{\infty} I_{\text {in }} r d r},
$$

where $\alpha_{0}$ is the linear absorption coefficient of the sample. Thus, by numerically solving Eqs. (1)-(6) and fitting to the experimental transmission data, the nonlinear absorption parameters $\beta_{\text {eff }}$ and $I_{\text {sat }}$ of the reverse saturable and saturable absorbers can be, respectively, determined. The fitting values are summarized in Table I. Analytical forms of the transmission for saturable absorbers and reverse saturable absorbers are given in Refs. 21 and 14, respectively.

In determining the nonlinear absorption parameters from PA signals, the following Tam's indirect PA generation model ${ }^{19}$ which describes the peak PA amplitude $\delta P$ was used

$$
\delta P \approx \frac{\gamma P s_{g} w^{2} I \alpha \mu_{s}^{2}}{T\left(R^{2} l_{g}+V_{r} / \pi\right) k_{s}},
$$

TABLE I. Summary of fitting values.

\begin{tabular}{lcc}
\hline \hline & Transmission & Photoacoustic \\
\hline PCBM $(\mathrm{T}=0.2)$ & $\beta_{\text {eff }}=1 \mathrm{~nm} / \mathrm{W}$ & $\beta_{\text {eff }}=0.8 \mathrm{~nm} / \mathrm{W}$ \\
$\operatorname{DODCI}(\mathrm{T}=0.2)$ & $\mathrm{I}_{\mathrm{sat}}=3.6 \mathrm{TW} / \mathrm{m}^{2}$ & $\mathrm{I}_{\text {sat }}=4 \mathrm{TW} / \mathrm{m}^{2}$ \\
PCBM $(\mathrm{T} \sim 0)$ & $\mathrm{N} / \mathrm{A}$ & $\beta_{\text {eff }}=0.6 \mathrm{~nm} / \mathrm{W}$ \\
$\operatorname{DODCI}(\mathrm{T} \sim 0)$ & $\mathrm{N} / \mathrm{A}$ & $\mathrm{I}_{\mathrm{sat}}=4 \mathrm{TW} / \mathrm{m}^{2}$ \\
CBS $(\mathrm{T}=0.01)$ & $\beta_{\text {eff }}=1.2 \mathrm{~nm} / \mathrm{W}$ & $\mathrm{N} / \mathrm{A}$ \\
\hline \hline
\end{tabular}

where $\gamma$ is the ratio of the specific heat, $P$ is the pressure inside the cell before laser irradiation, $w$ is the laser beam radius, $s_{g}$ is the effective thermal length of the coupling media, $\mu_{s}$ is the sample thermal diffusion length, $l_{g}$ is the coupling media thickness, $\alpha$ is the absorption coefficient, $I$ is the incident light intensity, $T$ is the absolute temperature, $R$ is the effective radius of the coupling media cross-section, $V_{r}$ is the residual volume in the PA cell, and $k_{s}$ is the sample thermal conductivity. For a nonlinear absorber, Eq. (7) can be modified to have the following form:

$$
\delta P \approx \frac{\gamma P s_{g} w^{2} I \mu_{a} \mu_{s}^{2}}{T\left(R^{2} l_{g}+V_{r} / \pi\right) k_{s}},
$$

where $\mu_{a}$ is given in Eqs. (2) and (3) for reverse saturable and saturable absorbers, respectively. In Z-scan configuration, the peak PA amplitude is a function of z-position given by

$$
\delta P \approx \frac{\gamma P s_{g} w(z)^{2} I(z) \mu_{a}(z) \mu_{s}^{2}}{T\left(R^{2} l_{g}+V_{r} / \pi\right) k_{s}} .
$$

By assuming Gaussian's distribution of the pulse both in spatial and temporal domains, the laser incident energy can be expressed as

$$
E=\frac{\pi \sqrt{\pi} I w^{2} t_{p}}{2} .
$$

Substituting Eq. (10) into Eq. (9) yields

$$
\delta P \approx \frac{2 \gamma P s_{g} E \mu_{a}(z) \mu_{s}^{2}}{\pi \sqrt{\pi} t_{p} T\left(R^{2} l_{g}+V_{r} / \pi\right) k_{s}} .
$$

In the Z-scan configuration, the incident energy is kept constant. The normalized photoacoustic signal is given by

$$
P A_{\text {norm }}(z)=\frac{P A(z)}{P A\left(\frac{z}{z_{0}} \gg 1\right)}=\frac{\mu_{a}(z)}{\alpha_{0}} .
$$

Thus, by fitting the measured data to Eq. (12), the nonlinear parameters can be determined. The fitting parameters are summarized in Table I. It is evident from Table I that the fitting parameters obtained from optical transmission and photoacoustic measurements are comparable.

In addition, we carried out PAZ-scan measurements in opaque samples of the same materials as shown in Figs. 2(c) and 2(d). Due to the opacity of the samples, the transmitted energy was too low to be measured by the photodetector and therefore yielded no Z-scan signal. The fitting values for PA data of these samples are also given in Table I. The fit parameters are comparable to those obtained in transparent samples from both transmission and photoacoustic measurements. These measurements clearly demonstrate the advantage of photoacoustic method over transmission method in determining the nonlinear optical coefficients of non-transparent samples.

In order to demonstrate the advantage of the OPAZ-scan (i.e., measuring both transmission and photacoustic data, 
simultaneously), we scanned carbon-black suspension (CBS), which is a nonlinearly scattering sample (Figure 3). Interestingly, comparing Figures 3 and 2, OPAZ curves of CBS reveals a different characteristic than what is expected from typical reverse saturable and saturable absorbers. Reverse saturable absorbers show decrease in transmission and increase in acoustic signal as the sample moves toward the focal point, while saturable absorbers show the opposite trend. Carbon black suspension, however, shows a decrease in the transmission and non-characteristic acoustic signals, as it is moved closer to the focal point. This observation suggests that the well-known optical limiting found in carbon-black suspensions does not originate primarily from nonlinear absorption. In fact, it has been shown that the nonlinear transmission of CBS for nanosecond pulses arises mostly from nonlinear scattering. ${ }^{22}$

It is obvious from Figure 3 that the $\mathrm{Z}$-scan curve alone is insufficient to determine the mechanisms behind the nonlinear reduction in transmission. For instance, an incorrect conclusion could be drawn because the Z-scan curves obtained from nonlinear scattering samples are similar to that obtained from reverse saturable absorbers. On the other hand, measuring the photoacoustic signal alone also is not possible to draw any conclusions. The fluctuation in the acoustic signal can be attributed to background noise in the acoustic detection created by nonlinear scattering process. Nevertheless, because the PAZ-scan curve does not show any characteristic sign of nonlinear absorption, the nonlinear parameter obtained from fitting Z-scan curve (Table I) can be mostly attributed to a nonlinear scattering process.

Since we found that nonlinear scatters give only a deterministic Z-scan curve, it might be possible to detect small nonlinear absorption signal from a highly nonlinear scattering system. In order to test this hypothesis, the OPAZ-scan measurement was done with a thick carbon black suspension in methanol mixed with small amount of saturable absorber dye, DODCI. The linear transmission of the mixture was $1 \%$. The OPAZ-scan data are plotted in Figure 4. Each data point is an average of 64 data samples from an oscilloscope. Such a high

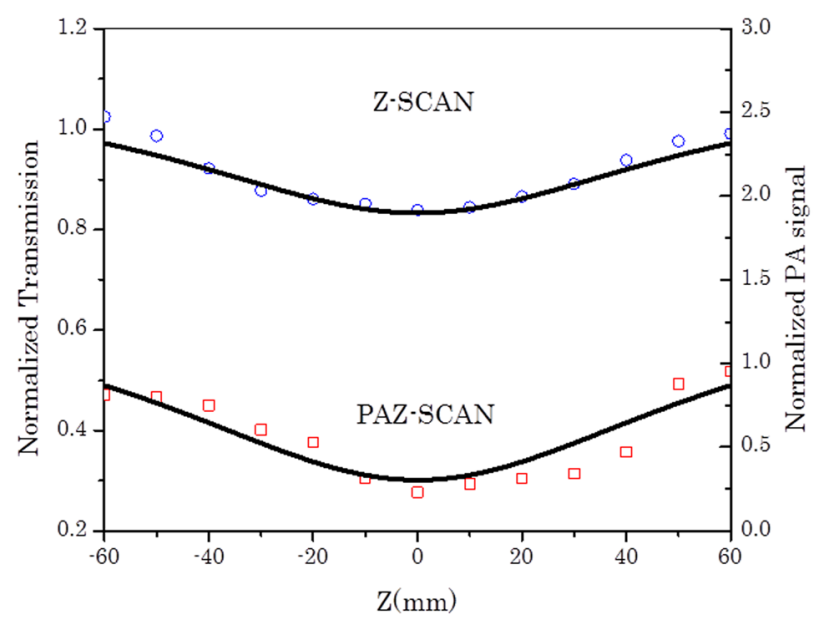

FIG. 4. OPAZ-scan curves of carbon-black dispersion in methanol mixed with saturable dyes DODCI. The linear transmission is $1 \%$ at $532 \mathrm{~nm}$ and Ein is $55 \mu \mathrm{J}$. The solid black lines are fitted curves. Open circles and rectangles are Z-scan and PAZ-scan data, respectively. volume of data samples was required due to high background noise.

From the OPAZ-scan data, the mixture shows valleys in both Z-scan and PAZ-scan curves. The Z-scan signal of the mixture might arise from the combination of nonlinear scattering and saturable absorption process. However, because there was only small amount of nonlinear absorbers present in the system, the nonlinear scattering dominated the Z-scan data, and as a result, the reduction in transmission was observed as the sample was moved toward the focal point. In contrast, unlike pure carbon black suspension, this mixture gave a characteristic PAZ-scan signal of saturable absorbers that was intentionally added. From this experiment, it is clear that our OPAZ-scan technique can detect nonlinear absorption even in the system with nonlinear scattering. The fitting value for Z-scan is $\beta$ eff of $0.95 \mathrm{~nm} / \mathrm{W}$, using Eqs. (1)-(6). For the PAZ-scan data, Eq. (12) was used for fitting and the fitting value is Isat of $0.5 \mathrm{TW} / \mathrm{m}^{2}$. Having two sets of data available by running OPAZ-scan, the nonlinear processes within a given system can be deduced. In the case of our mixture, by looking at the photoacoustic signal, it can be determined that there is saturable absorption present in the system. The Z-san curve, on the other hand, suggests that there is another nonlinear process in the system that causes the Z-scan curve to have a flipped shape from a typical system with pure saturable absorbers. Such a process which is responsible for the valley curve of the z-scan cannot be a nonlinear absorption process because otherwise it would be reflected in the PA signal as well. It can be seen from the previous paragraph that for pure nonlinear absorber system, PAZ-scan and Z-scan are to some extent mirror images of each other. Nonetheless, in our case, nonlinear scattering process is responsible in the flipped Z-scan behavior. This finding demonstrates that the simultaneous measurement of both acoustic and optical signals is advantageous in accomplishing a better insight into the nonlinear optical phenomenon.

In summary, we have developed a measurement technique, by combining optical Z-scan and photoacoustic Zscan, called OPAZ-scan. We have demonstrated that this technique benefits from the advantages of both measurements. With this system, nonlinear absorption of both optically light and dark sample can be measured. Furthermore, It was also found that the simultaneous measurement of the optical and PA Z-scan signals gives a better insight into optical nonlinearity, especially ones with mixed nonlinear scattering and absorption.

J.T. acknowledges University of Central Florida start-up funding for the completion of this work. R.P. thanks Raman Research Institute, India for sabbatical leave.

${ }^{1}$ D. J. Harter, M. L. Shand, and Y. B. Band, J. Appl. Phys. 56, 865-868 (1984).

${ }^{2}$ T. Xia, D. J. Hagan, A. Dogariu, A. A. Said, and E. W. Van Stryland, Appl. Opt. 36, 4110-4122 (1997).

${ }^{3}$ C. W. Spangler, J. Mater. Chem. 9, 2013-2020 (1999).

${ }^{4}$ G.-K. Lim, Z.-L. Chen, J. Clark, R. G. S. Goh, W.-H. Ng, H.-W. Tan, R. H. Friend, P. K. H. Ho, and L.-L. Chua, Nature Photon. 5, 554-560 (2011).

${ }^{5}$ J. D. Bhawalkar, N. D. Kumar, C.-F. Zhao, and P. N. Prasad, J. Clin. Laser Med. Surg. 15, 201-204 (1997). 
${ }^{6}$ X. Huang, W. Qian, I. H. El-Sayed, and M. A. El-Sayed, Laser Surg. Med. 39, 747-753 (2007).

${ }^{7}$ T. F. Boggess, S. C. Moss, I. W. Boyd, and A. L. Smirl, Opt. Lett. 9, 291293 (1984).

${ }^{8}$ D. G. McLean, R. L. Sutherland, M. C. Brant, D. M. Brandelik, P. A. Fleitz, and T. Pottenger, Opt. Lett. 18, 858-860 (1993).

${ }^{9}$ A. J. Taylor, R. B. Gibson, and J. P. Roberts, Opt. Lett. 13, 814-816 (1988).

${ }^{10}$ E. Eva and K. Mann, Appl. Phys. A: Mater. Sci. Process. 62, 143-149 (1996).

${ }^{11}$ M. Bass, E. W. Van Stryland, and A. F. Stewart, Appl. Phys. Lett. 34, 142-144 (1979).

${ }^{12}$ Y. Bae, J. J. Song, and Y. B. Kim, Appl. Opt. 21, 35-40 (1982).

${ }^{13}$ C. Ferrante, R. Signorini, A. Feis, and R. Bozio, Photochem. Photobiol. Sci. 2, 801-807 (2003).

${ }^{14}$ M. Sheik-Bahae, A. A. Said, T. H. Wei, D. J. Hagan, and E. W. Van Stryland, IEEE J. Quantum Electron. 26, 760-769 (1990).
${ }^{15}$ P. Chantharasupawong, R. Philip, T. Endo, and J. Thomas, Appl. Phys. Lett. 100, 221108 (2012).

${ }^{16}$ R. Philip, P. Chantharasupawong, H. Qian, R. Jin, and J. Thomas, Nano Lett. 12, 4661-4667 (2012).

${ }^{17}$ P. Chantharasupawong, R. Philip, N. T. Narayanan, P. M. Sudeep, A. Mathkar, P. M. Ajayan, and J. Thomas, J. Phys. Chem. C 116, 2595525961 (2012).

${ }^{18}$ C. S. Yelleswarapu and S.-R. Kothapalli, Opt. Express 18, 9020-9025 (2010).

${ }^{19}$ A. C. Tam, Rev. Mod. Phys. 58, 381-431 (1986).

${ }^{20}$ E. W. V. Stryland, M. Sheik-Bahae, E. M. G. Kuzyk, and C. W. Dirk, Characterization Techniques and Tabulations for Organic Nonlinear Materials (Marcel Dekker, Inc., 1998), pp. 655-692.

${ }^{21}$ B. Gu, Y.-X. Fan, J. Wang, J. Chen, J. Ding, H.-T. Wang, and B. Guo, Phys. Rev. A 73, 065803 (2006).

${ }^{22}$ K. Mansour, M. J. Soileau, and E. W. V. Stryland, J. Opt. Soc. Am. B 9, 1100-1109 (1992). 